\title{
Operation and Performance of the ATLAS Silicon Micro-strip Tracker
}

\author{
Y. Pylypchenko for the ATLAS Collaboration
}

\begin{abstract}
The SemiConductor Tracker (SCT) is the key precision tracking device in ATLAS, made from silicon microstrip detectors processed in the planar p-in-n technology. The completed SCT has been installed inside the ATLAS experimental hall since 2007 and has been operational since then.

In this paper the current status of the SemiConductor Tracker is reviewed, including results from the latest data-taking periods in 2009 and 2010, and from the detector alignment. The main emphasis is given to the performance of the SemiConductor Tracker with the LHC in collision mode and to the performance of individual electronic components.
\end{abstract}

\section{INTRODUCTION}

ATLAS [1] is one of the two large general purpose detectors built at the Large Hadron Collider (LHC) [2] to explore physics at TeV scale. It consists of several detector subsystems. Closest to the interaction point is the Inner Detector, the charged particle tracker immersed in a $2 \mathrm{~T}$ axial magnetic field provided by a superconducting solenoid magnet. The Inner Detector comprises two silicon detectors, the Pixel Detector and the SemiConductor Tracker, and a Transition Radiation Tracker. The liquid argon electromagnetic calorimeters surround the Inner Detector. The electromagnetic calorimeters are, in turn, surrounded by hadronic calorimeters. The outermost system of the ATLAS detector is the Muon Spectrometer which relies on a set of massive superconducting air-core toroid magnets.

ATLAS started collision data taking in December 2009. Integrated luminosity recorded in 2009 during stable beams and for proton-proton collisions at $900 \mathrm{GeV}$ centre-of-mass energy equals $12 \mu \mathrm{b}^{-1}$. The LHC collision energy was increased to the unprecedented $7 \mathrm{TeV}$ in March 2010 and ATLAS recorded $45 \mathrm{pb}^{-1}$ for $7 \mathrm{TeV}$ proton-proton collisions during 2010. In addition to that $9.17 \mu \mathrm{b}^{-1}$ of data were taken during heavy ion $(\mathrm{Pb})$ collision run at $2.76 \mathrm{TeV}$ centre-of-mass energy per nucleon at the end of 2010 .

\section{THE ATLAS SEMICONDUCTOR TRACKER}

The SemiConductor Tracker (SCT) forms the intermediate tracking layers of the ATLAS Inner Detector (see Fig. II). With $61 \mathrm{~m}^{2}$ of an active silicon area and 6.3 million readout channels it provides precision measurements in the radial range $30<r<56 \mathrm{~cm}$ for charged particle tracks with

Y. Pylypchenko is with the Department of Physics, University of Oslo, Postbox 1048, 0316 Oslo, Norway, e-mail: yuriy.pylypchenko@fys.uio.no. $|\eta|<2.5^{1}$. It is made up of a barrel and two endcaps

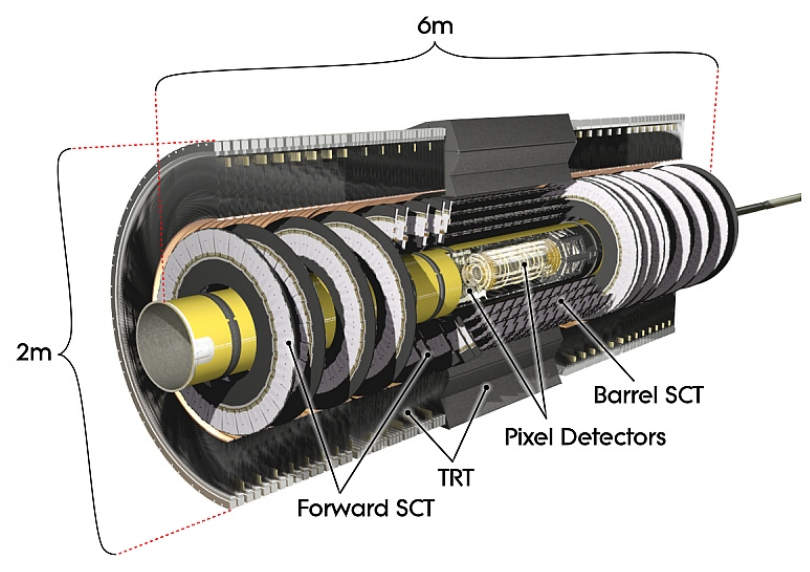

Fig. 1. A schematic view of the ATLAS Inner Detector.

comprising 4088 silicon micro-strip modules. The 2112 barrel modules are arranged to form four concentric layers covering pseudorapidity range $|\eta|<1.1$ to 1.4 depending on the layer. The 1976 endcap modules, arranged in nine disks in each of the endcaps, extend the $\eta$ coverage to $|\eta|<2.5$. All barrel modules are identical [3], whereas the endcap modules come in four types differing primarily in shape [4].

The general layout of the SCT module represents the two pairs of identical, single-sided silicon micro-strip sensors each with 768 active p-strips on n-type silicon bulk. The pitch is $80 \mu \mathrm{m}$ for barrel modules and 57-94 $\mu \mathrm{m}$ for endcap modules, while the sensor thickness is $285 \mu \mathrm{m}$. For most of the modules each pair of sensors is wire-bonded, forming $126 \mathrm{~mm}$ long strips. The sensors are glued back-to-back on a thermal pyrolitic graphite baseboard and rotated by a $40 \mathrm{mrad}$ stereo angle to provide a $\mathrm{z}$-measurement capability. This module design allows for intrinsic strip efficiency greater that $99 \%$ and a space-point resolution of $17 \mu \mathrm{m}$ in bending $r-\phi$ plane and $580 \mu \mathrm{m} \mathrm{z}$-direction.

To limit the radiation damage effects, such as reverse annealing and leakage current, and to decrease the noise levels, the SCT detector is cooled down by evaporative cooling

${ }^{1}$ ATLAS uses a right-handed coordinate system with its origin at the nominal interaction point. The counterclockwise beam direction defines the $\mathrm{z}$-axis, the positive $\mathrm{y}$-axis points towards the center of the LHC ring and the positive $y$-axis points upwards. Cylindrical coordinates $(r, \phi)$ are used in the transverse plane with respect to the beam and the pseudorapidity $\eta$ is defined in terms of the polar angle $\theta$ as $\eta=-\ln \tan (\theta / 2)$. 
system which uses perfluorinated propan (C3F8) with nominal operating temperature at $-25^{\circ} \mathrm{C}$ [5]. Cooling channels contact each of the SCT modules. The module baseboard serves as the thermal conductor to remove the heat generated by the readout electronics and sensors.

The signals from the strips are processed in the front-end ABCD3TA ASICs [6], realized in the radiation hard DMILL technology and working in the binary readout mode in which the signal from the silicon strip is amplified, shaped and discriminated yielding only hit or no-hit information.

The communication between each SCT module and the off-detector data acquisition system is realized via individual optical links based on Vertical Cavity Surface Emitting Lasers (VCSEL) and epitaxial Si p-i-n diodes [7]. Each module is equipped with two data links, operation at $40 \mathrm{Mbits} / \mathrm{s}$, to transfer data to the off-detector readout electronics. The optical links are also used to send control signal from counting room to the front-end electronics. The main control and data handling is performed off-detector by the readout driver [8].

Each module is served by its own independent power supply, with a low voltage power supply for the on-detector electronics and optical communication components, and a separate high voltage card providing the bias voltage for the module sensors. The typical depletion voltage is approximately $60 \mathrm{~V}$, while the default reverse bias voltage is $150 \mathrm{~V}$, at which the SCT is operated when there are stable beams. In the absence of stable beams the bias voltage is kept at $50 \mathrm{~V}$ for safety reasons.

The completed SCT has been installed inside the ATLAS experimental hall in 2007 and has been operational since then. Extensive commissioning with calibration data and cosmic ray events were performed to tune the system and determine its performance. After the commissioning phase the SCT was ready for the first proton-proton collision run. Since the LHC turn-on in 2009 the SCT has proven to be reliable and efficient.

\section{OpERATION OF THE ATLAS SEMICONDUCTOR TRACKER}

The SCT successfully operated during 2009-2010 data taking including the period of the LHC heavy ion running. It provided zero contribution to ATLAS dead-time and its small contribution to overall data taking inefficiencies were only due to ramping up and ramping down of the high voltage of the modules during beam injection. The whole detector was well synchronized with the trigger signal. Typically $99.3 \%$ of the total of 4088 modules were operational. A summary of the

\begin{tabular}{l|ccccc}
$\begin{array}{l}\text { Disabled Readout } \\
\text { Components }\end{array}$ & Endcap A & Barrel & Endcap C & SCT & $\begin{array}{c}\text { Fraction } \\
(\%)\end{array}$ \\
\hline Disabled Modules & 5 & 10 & 15 & 30 & 0.73 \\
Disabled Chips & 5 & 24 & 4 & 33 & 0.07 \\
Masked Strips & 3,364 & 3,681 & 3,628 & 10,673 & 0.17 \\
\hline $\begin{array}{l}\text { Total Disabled } \\
\text { Detector Region }\end{array}$ & & & & & 0.97 \\
\hline
\end{tabular}

Fig. 2. A summary of SCT components excluded from the configuration during data taking in 2010 .

disabled SCT modules and chips as well as masked strips during the data taking in 2010 is shown in Fig. 2. 13 modules were affected by one cooling loop in the endcap $\mathrm{C}$ which was not working due to a leak. The rest of the modules were not operational due to connection problems. The number of masked strips include the defective and noisy strips.

There were problems with the off-detector optical transmitters sending clock and control signals to modules (TX plugins), which were resulting in unexpected failures during the running period. This problem is understood to be due to the ingress of humidity into the VCSEL. Since redundancy can be used for optical connections a TX plugin failure does not necessarily imply a gap in the acceptance. There are also replacement transmitters with different, more robust VCSELs.

The operation of the evaporating cooling system has been of a special attention since a number of problems in the past. During 2009-2010 data taking the overall cooling system performance was smooth and efficient. The SCT operated with the temperature of $-7^{\circ} \mathrm{C}$ at a silicon surface in the endcaps and $-2^{\circ} \mathrm{C}$ in the barrel, except for the outermost barrel layer which operated with $+4.5^{\circ} \mathrm{C}$ at a silicon surface. The SCT barrel operated at higher temperatures in order to compensate for the failed thermal enclosure heaters and ensure thermal neutrality of the SCT volume compared to that of the Transition Radiation Tracker.

In the following various aspects of the SCT operation demonstrating the performance during 2009-2010 are described.

\section{Performance of the Semiconductor Tracker}

\section{A. Noise level}

The noise occupancy of the SCT detector is measured using two methods: noise occupancy test and response curve test. In the first case the noise level is determined by measuring the noise occupancy as a function of threshold using random triggers. A fit to this distribution allows for estimation of the noise. Fig. 3 shows the noise occupancy measured at nominal $1 \mathrm{fC}$ threshold with the noise occupancy test. As one can see, it is below the design specification of $5 \times 10^{-4}$. The correspondent noise distributions are shown in Fig. 4.

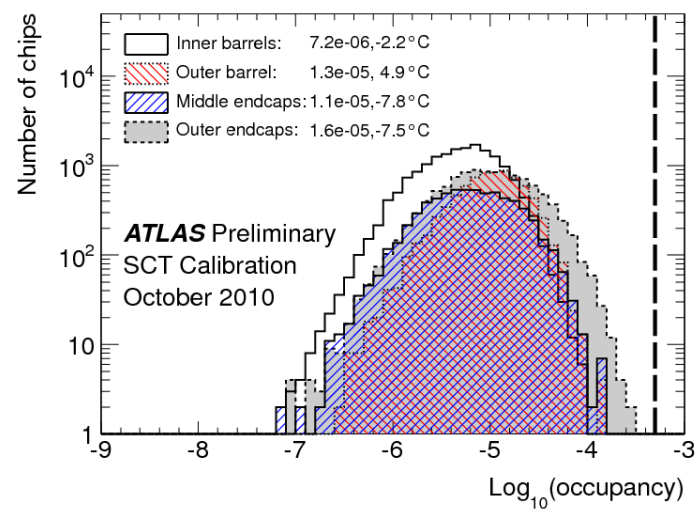

Fig. 3. The distribution of the noise occupancy for each chip split up for the different parts of the SCT. Dashed line shows the TDR specification. The temperatures of the silicon sensors shown on the plots are derived from the measured hybrid temperatures using a correction determined from simulation. 


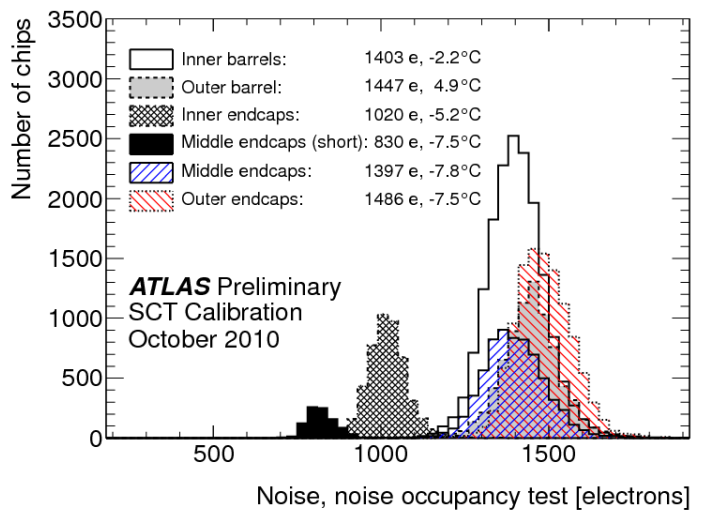

Fig. 4. The distribution of the input noise estimated from noise occupancy test in the different parts of the SCT. The temperatures of the silicon sensors shown on the plots are derived from the measured hybrid temperatures using a correction determined from simulation.

In the second method the occupancy at different thresholds is measured by injecting various known charges. For each value of injected charge the threshold is scanned over the entire range and a complementary error function is fitted to the average occupancy. The variance of this distribution characterizes the noise. Both methods yield compatible resutls.

\section{B. Hit efficiency}

Fig. 5 shows the number of hits per expected hit which represents the intrinsic silicon strip efficiency. It is evaluated for two types of tracks: SCT standalone and combined Inner Detector tracks, both satisfying the requirement of a transverse momentum above $1 \mathrm{GeV}$. For standalone tracks at least 7 SCT hits are required, excluding the hit under test for efficiency, while for combined tracks at least 6 SCT hits are required. The values obtained for both types of tracks are compatible being of about $99.8 \%$ and $99.9 \%$ for the strips in SCT endcaps and SCT barrel respectively. This is higher than the design requirement of $99 \%$ for the intrinsic silicon strip efficiency.

\section{Strip occupancy}

A comparison of the number of hit strips per module side for $900 \mathrm{GeV}$ collision data and Monte Carlo simulated events is plotted in Fig. 6. The plot contains all SCT modules both from barrel and endcaps. Both distributions are normalized by number of events. Good agreement over several orders of magnitude can be seen between the data and Monte Carlo distributions. The discrepancy in the first bin is due to a lower level of noise in the simulation than in data. Noise in the simulation is appropriate for readout of one bunch crossing while the data were collected over three bunch crossings leading to a higher noise level.

\section{Lorentz angle}

The Lorentz angle is the angle by which charge carriers in the silicon sensor drift in the presence of the magnetic field. It depends on magnetic field strength, module temperature and
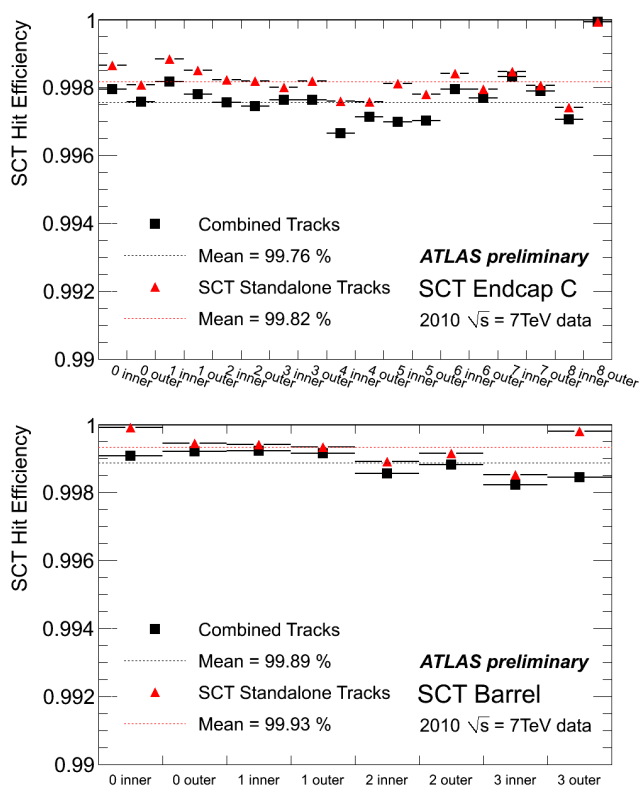

Fig. 5. The intrinsic silicon strip efficiency is shown for tracks measured in the SCT endcap C (upper plot) and the SCT barrel (lower plot). The data used are from $7 \mathrm{TeV}$ collision run taken in September 2010.

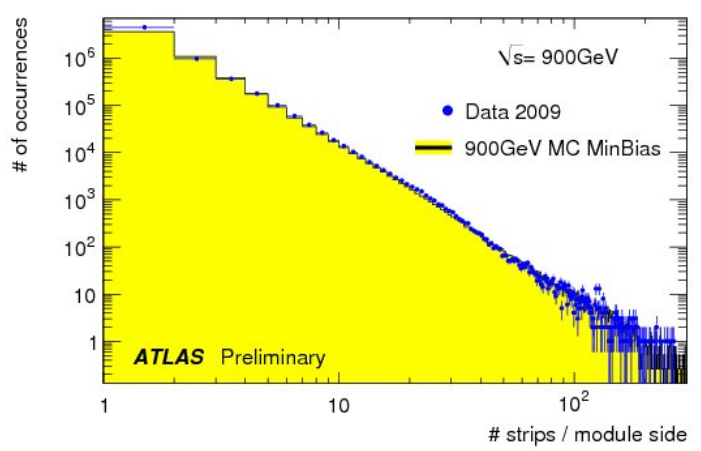

Fig. 6. The number of strips per module side from a $900 \mathrm{GeV}$ run compared with a $900 \mathrm{GeV}$ minimum bias Monte Carlo sample.

bias voltage. The Lorentz angle can be obtained by determining the track incidence angle in the plane perpendicular to the magnetic field which yields the minimum average cluster width (Fig. 7). This angle equals the Lorentz angle and is zero in the absence of the magnetic field. Fig. 8 shows the measured Lorentz angle in each layer of the SCT barrel. The extracted values and their statistical errors are shown for $900 \mathrm{GeV}$ collision data and cosmic ray data collected with the SCT operating under nominal conditions. The model prediction that is also shown on the plot takes into account the fact that the layers are not operated at the same temperature. The measured values can be seen to lie within about $1 \sigma$ of the model prediction, although systematically above it. Further investigations are ongoing. 


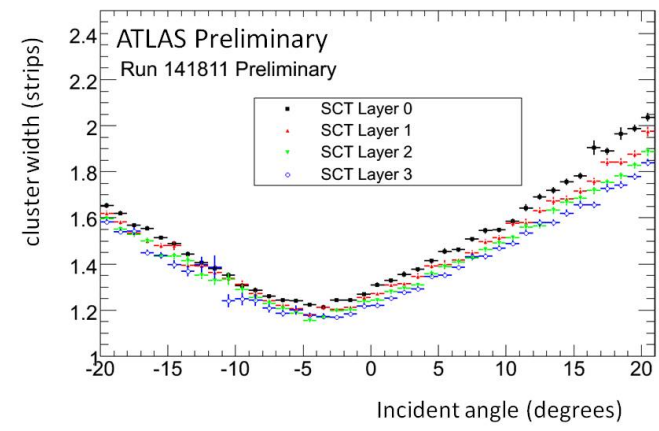

Fig. 7. The SCT cluster size (in strips) plotted as a function of the track incidence angle for the four layers in the SCT barrel.

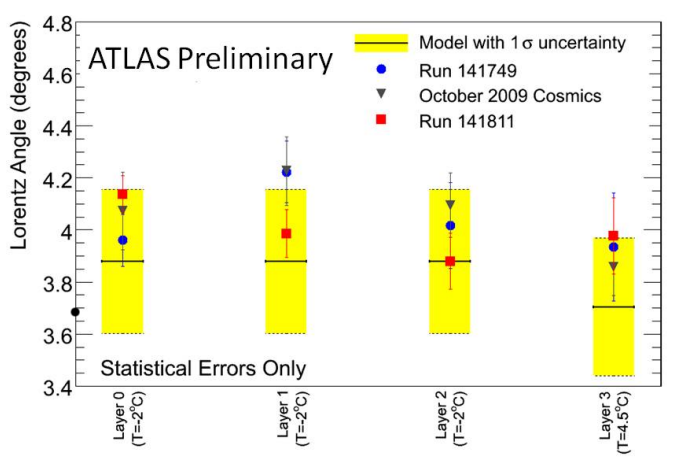

Fig. 8. The values of the Lorentz angles extracted for four SCT barrel layers.

\section{E. Alignment}

The tracking and invariant mass reconstruction performance depend on the precision of the positioning of the silicon modules. The alignment procedure is applied to accurately determine their position and correct for systematic effects that could bias the tracking. Fig. 9 shows an impressive agreement between the measured $K_{S}^{0}$ mass using $900 \mathrm{GeV}$ collision data and the world average value [9], indicating that the performance of the alignment was very good already in 2009.

In 2010 the SCT alignment approached the ideal case. Fig. 10 shows the $\mathrm{x}$ residual distribution for $7 \mathrm{TeV}$ collision data compared to Monte Carlo simulation with a perfectly aligned detector. The residuals are calculated as the difference between the measured hit coordinate and the expected position obtained from track extrapolation. Tracks used for the calculation were selected to have transverse momentum greater than $2 \mathrm{GeV}$ and at least 6 silicon hits. As can be seen the data are in a very good agreement with the perfectly aligned detector simulation.

\section{CONCLUSIONS}

The ATLAS SemiConductor Tracker is fully operational and is being successfully used in ATLAS data taking contributing to physics results.

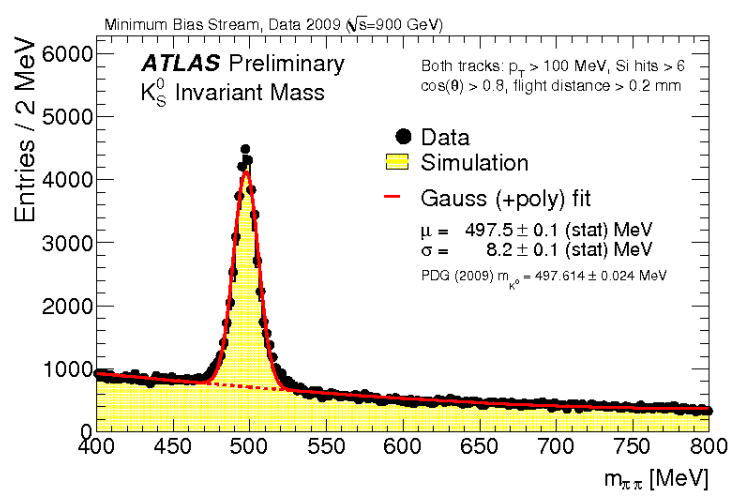

Fig. 9. The invariant mass of pion pairs from $K_{s}^{0} \rightarrow \pi^{+} \pi^{-}$decay reconstructed from $900 \mathrm{GeV}$ collision data using the information from the entire ATLAS Inner Detector.
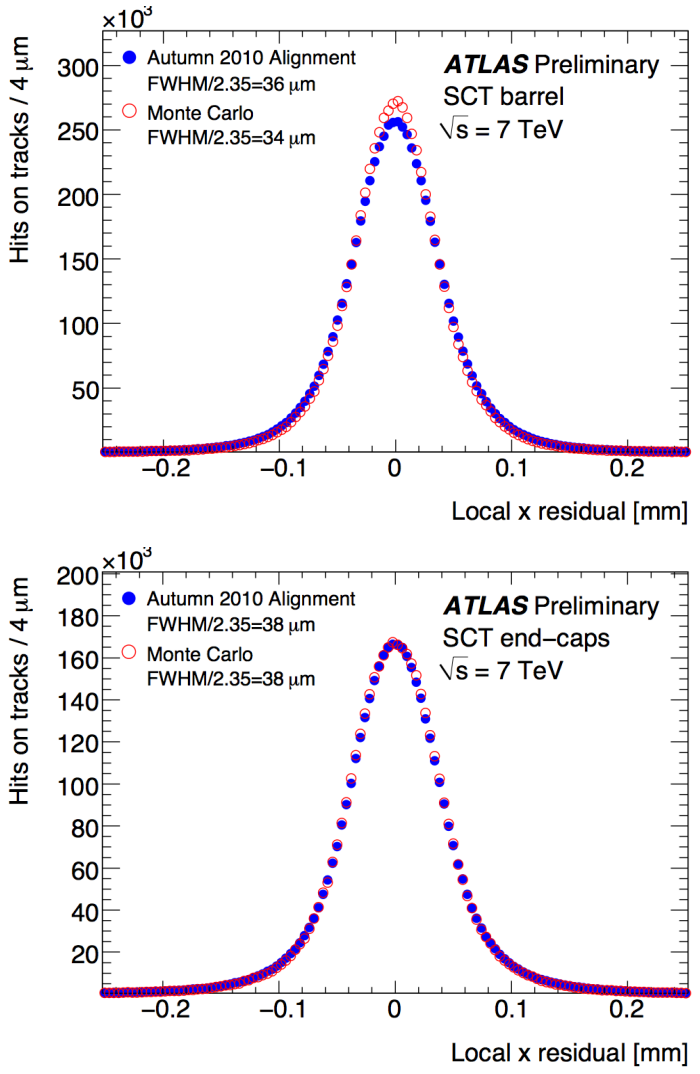

Fig. 10. Distribution of the local $\mathrm{x}$ unbiased residuals of the SCT barrel modules (upper plot) and the SCT endcap modules (lower plot). Full blue circles show the data residuals after the detector alignment, and the open red circles show the residuals using Monte Carlo with a perfectly aligned detector (normalized to the number of entries in the data distribution).

It demonstrated an excellent performance in 2009 and 2010: $99.3 \%$ of the SCT modules were operational, the noise occupancy level and hit efficiency exceeded the design specifications and the alignment being very close to ideal allowing for on-line track reconstruction and invariant mass determination. Measurements of the quantities such as occupancy and Lorentz angle demonstrate the understanding of the detector and allow for fine tuning of its Monte Carlo simulation. 


\section{REFERENCES}

[1] G. Aad et al., The ATLAS Experiment at the CERN Large Hadron Collider, JINST 3 (2008) S08003

[2] L. Evans and P. Bryant, LHC Machine, JINST, 3 (2008) S08001

[3] A. Abdesselam et al., The barrel modules of the ATLAS semiconductor tracker, Nucl. Instrum. Meth. A568 (2006) p. 642-671

[4] A. Abdesselam et al., The ATLAS semiconductor tracker end-cap module, Nucl. Instrum. Meth. A575 (2007) p. 353-389

[5] D. Attree et al., The evaporative cooling system for the ATLAS inner detector, JINST 3 (2008) P07003

[6] F. Campabadal et al., Design and performance of the ABCD3TA ASIC for readout of silicon strip detectors in the ATLAS semiconductor tracker, Nucl. Instrum. Meth. A552 (2005) p. 292-328

[7] A. Abdesselam et al., The optical links of the ATLAS SemiConductor Tracker", JINST 2 (2007) P09003

[8] A. Abdesselam et al., The data acquisition and calibration system for the ATLAS Semiconductor Tracker, JINST 3 (2008) P01003

[9] C. Amsler et al. (Particle Data Group), Review of Particle Physics, Phys. Lett. B667 (2008) 1 